\title{
Structural characterization of the electric field-induced ferroelectric phase in $\mathrm{Na0.5Bi0.5TiO3-KNbO3}$ ceramics
}

DOI:

10.1016/j.jeurceramsoc.2016.06.022

\section{Document Version}

Accepted author manuscript

Link to publication record in Manchester Research Explorer

\section{Citation for published version (APA):}

Wang, G., Hall, D., Li, Y., Murray, C., \& Tang, C. (2016). Structural characterization of the electric field-induced ferroelectric phase in $\mathrm{Na} \mathrm{Bi} \mathrm{TiO}_{3}-\mathrm{KNbO}_{3}$ ceramics. Journal of the European Ceramic Society, 36(16), 20152021. https://doi.org/10.18 \$6/j.jeurcelamsoc.2016.06.022

\section{Published in:}

Journal of the European Ceramic Society

\section{Citing this paper}

Please note that where the full-text provided on Manchester Research Explorer is the Author Accepted Manuscript or Proof version this may differ from the final Published version. If citing, it is advised that you check and use the publisher's definitive version.

\section{General rights}

Copyright and moral rights for the publications made accessible in the Research Explorer are retained by the authors and/or other copyright owners and it is a condition of accessing publications that users recognise and abide by the legal requirements associated with these rights.

\section{Takedown policy}

If you believe that this document breaches copyright please refer to the University of Manchester's Takedown Procedures [http://man.ac.uk/04Y6Bo] or contact uml.scholarlycommunications@manchester.ac.uk providing relevant details, so we can investigate your claim.

\section{OPEN ACCESS}




\title{
Structural characterization of the electric field-induced ferroelectric phase in $\mathrm{Na}_{0.5} \mathrm{Bi}_{0.5} \mathrm{TiO}_{3}-\mathrm{KNbO}_{3}$ ceramics
}

\author{
Ge. Wang ${ }^{1}$, David. Hall ${ }^{1}$, Yizhe. $\mathrm{Li}^{1}$, Claire. Murray ${ }^{2}$ and Chiu. Tang ${ }^{2}$ \\ ${ }^{1}$ School of Materials, University of Manchester, Manchester, M13 9PL, UK \\ ${ }^{2}$ Diamond Light Source Ltd, Diamond House, Harwell Science and Innovation Campus, \\ Didcot, Oxfordshire, OX11 ODE, UK
}

\begin{abstract}
(1-x) $\mathrm{Na}_{0.5} \mathrm{Bi}_{0.5} \mathrm{TiO}_{3}-\mathrm{xKNbO}_{3}$ (NBT-xKN) ceramics, with $\mathrm{x}$ in the range 0.01 to 0.09 , were prepared by solid state reaction and conventional sintering. A progressive transformation from a nano-polar to a long-range ordered ferroelectric phase was observed after the application of a cyclic electric field with an amplitude of $5 \mathrm{kV} \mathrm{mm}^{-1}$. It is shown by analysis of the crushed ceramic pellets, using high resolution synchrotron x-ray diffraction, that the application the cyclic electric field induced an irreversible transformation from a pseudo-cubic nano-polar phase to a rhombohedral ordered ferroelectric phase having R3c symmetry. Furthermore, a systematic variation, from $89.6^{\circ}$ to $90^{\circ}$, was observed in the rhombohedral interaxial angle as the KN content increased from 1 to $9 \%$. By comparing the structural evidence with the ferroelectric polarization-electric field hysteresis loops, it is shown that the electric field-induced transformation is partially reversible for NBT-5KN and completely reversible or nonexistent for NBT-9KN.
\end{abstract}

Key words: lead-free, piezoelectric ceramics, phase transition, ferroelectric properties, synchrotron x-ray diffraction 


\section{Introduction}

Piezoelectric materials have been widely used in many applications for over 100 years due to their unique electro-mechanical properties. So far, the most popular and widely used piezoelectric ceramic is lead zirconate titanate (PZT), which is used in many applications such as actuators, sensors and transducers. ${ }^{1}$ Superior piezoelectric coefficients and polarization have been obtained for compositions close to the morphotropic phase boundary (MPB) between rhombohedral and tetragonal phases. However, to deal with contamination by lead/lead oxide in the environment, lead-free piezoelectric ceramics have been investigated extensively over the past few decades and are gradually moving towards practical device applications. ${ }^{2} \mathrm{Na}_{0.5} \mathrm{Bi}_{0.5} \mathrm{TiO}_{3}$ (NBT), a perovskite-type relaxor-ferroelectric ceramic, is considered to be a promising candidate due to its large remanent polarization and relatively high Curie temperature. ${ }^{3}$ Also further investigations have been done to analyse its structural phase transformations and electrical properties. ${ }^{4,5}$ However, in order to overcome its high coercive field ${ }^{6}\left(E_{c}=7.3 \mathrm{kV} \mathrm{mm}^{-1}\right.$ in single crystal form) and to make use of on MPB to achieve the best piezoelectric properties, many solid solutions have been created by incorporating NBT into binary or ternary systems.

For example, a large electric field-induced strain of $0.29 \%$ and a normalized $d_{33}{ }^{*}$ value of $488 \mathrm{pm} \mathrm{V}^{-1}$ were obtained for materials in the $\mathrm{Na}_{0.5} \mathrm{Bi}_{0.5} \mathrm{TiO}_{3}-\mathrm{SrTiO}_{3}$ (NBT-ST) system. ${ }^{7} \mathrm{~A}$ high strain value of $0.45 \%$ was attained under a field of $8 \mathrm{kV} \mathrm{mm}^{-1}$ for compositions near the MPB in the $\mathrm{Na}_{0.5} \mathrm{Bi}_{0.5} \mathrm{TiO}_{3}-\mathrm{BaTiO}_{3}-\mathrm{K}_{0.5} \mathrm{Na}_{0.5} \mathrm{NbO}_{3}$ (NBT-BT-KNN) system. Furthermore, a MPB was located in the ternary system $\mathrm{Na}_{0.5} \mathrm{Bi}_{0.5} \mathrm{TiO}_{3}-\mathrm{K}_{0.5} \mathrm{Bi}_{0.5} \mathrm{TiO}_{3}-\mathrm{KNbO}_{3}$ (NBT-KBT-KN), leading to a $\mathrm{d}_{33}$ value of $215 \mathrm{pC} \mathrm{N}^{-1}$ at a field of $4-5 \mathrm{kV} \mathrm{mm}^{-1}$. 9 The so-called 'giant' electric field-induced strains in NBT-based systems have been attributed to a field-induced phase transformation. ${ }^{10}$ For example, an electric field-induced phase transformation from rhombohedral to mixed phases of rhombohedral and tetragonal occurred under a field of $2 \mathrm{kV} \mathrm{mm}^{-1}$ in the NBT-xKBT system, ${ }^{11}$ and a pseudo-cubic to tetragonal phase transformation took place under $3 \mathrm{kV} \mathrm{mm}^{-1}$ in the NBT-xBT system. ${ }^{12}$

$\mathrm{KNbO}_{3}(\mathrm{KN})$ is a well-known ferroelectric compound and is considered to be a useful component of lead-free piezoelectric ceramics due to its high Curie temperature, $T_{c}=$ $435{ }^{\circ} \mathrm{C}$, and large spontaneous polarization, $\mathrm{P}_{\mathrm{s}}=0.30 \mathrm{C} \mathrm{m}^{-2} .{ }^{13}$ In common with barium titatane, it has a sequence of phase transitions at temperatures of approximately $-50{ }^{\circ} \mathrm{C}$, $225^{\circ} \mathrm{C}$ and $435^{\circ} \mathrm{C}$ from rhombohedral to orthorhombic to tetragonal to cubic. ${ }^{13,14}$ By using the MPB between a ferroelectric and anti-ferroelectric phase, $\mathrm{KNbO}_{3}$ can be combined with $\mathrm{NaNbO}_{3}$ to form one of the best known lead-free piezoelectric solid solutions, $\mathrm{K}_{0.5} \mathrm{Na}_{0.5} \mathrm{NbO}_{3}{ }^{15}$ For the NBT-xKN system, a MPB between rhombohedral and pseudo-cubic phases was identified at approximately $\mathrm{x}=0.05$ by Fan. ${ }^{16,17} \mathrm{~A}$ further investigation by Hiruma ${ }^{18}$ suggested that the pseudo-cubic phase actually has a tetragonal structure. According to a different report, by Pisitpipathsin, ${ }^{19}$ a mixed-phase of both rhombohedral and orthorhombic was observed at $x=0.05$. However, the combination of small rhombohedral distortion and peak broadening gives rise to overlapping of diffraction peaks in the mixed-phase region, which hinders the unambiguous identification of crystal structure without more detailed full-pattern refinement. Recently, promising electro-caloric 
properties were reported for NBT-0.06KN but there was no investigation of the phase composition in those studies. ${ }^{20,21}$

It was shown in a previous publication ${ }^{22}$ that NBT-xKN ceramics with $x=0.02$ to 0.08 appear to have a pseudo-cubic structure in the as-sintered state but also exhibit well-formed P-E hysteresis loops for certain compositions, indicating the presence of a ferroelectric phase. Furthermore, it was found that the application of a high electric field during the measurement procedure induced a transformation to a ferroelectric phase having rhombohedral symmetry with a strong degree of preferred orientation. Also, ferroelectric and dielectric properties were investigated to provide an indication of the reversibility of the electric field-induced phase transformation.

The aim of the present study was to determine unambiguously the effect of a high electric field on the crystalline phases present in NBT-xKN ceramics, making use of high resolution synchrotron X-ray powder diffraction (SXPD) and full-pattern refinement to evaluate their crystallographic parameters. Potential issues associated with preferred orientation and anisotropic lattice strain induced by poling ${ }^{22}$ have been addressed by the use of crushed pellets during the analysis. It was shown previously that such a method is a valid approach to providing a stress-free and randomly-oriented powder, which is a suitable subject for full-pattern refinement procedures. ${ }^{23,24}$ Garg et al. observed that the process of mechanical crushing induced a slight change in the XRD peak profiles of NBT-BT ceramics, which was similar to but not as pronounced as the effect of electrical poling. ${ }^{23}$ In the present case, we assume that such an effect, if present, did not have a significant influence on the crystal structure of the poled ceramics.

\section{Experimental Methods}

Analytical-grade powders: $\quad \mathrm{Na}_{2} \mathrm{CO}_{3}(99.8 \%), \quad \mathrm{K}_{2} \mathrm{CO}_{3}(99 \%), \quad \mathrm{Bi}_{2} \mathrm{O}_{3}(99 \%), \quad \mathrm{TiO}_{2}(99 \%)$, $\mathrm{Nb}_{2} \mathrm{O}_{3}(99 \%)$ were used as raw materials to prepare (1-x).NBT-x.KN ( $\mathrm{x}=0.01,0.03,0.05$, 0.09 ) ceramics. The mixed powders were milled for $24 \mathrm{~h}$ in propan-2-ol using zirconia milling balls and calcined for $10 \mathrm{~h}$ at $900{ }^{\circ} \mathrm{C}$ to accomplish the solid state reaction. The as-calcined powders were milled again for $24 \mathrm{~h}$ to break down the hard agglomerates. The final dry powders were uniaxially pressed into $6.5 \mathrm{~mm}$ diameter pellets under a pressure of $150 \mathrm{MPa}$ and sintered at $1180{ }^{\circ} \mathrm{C}$ for 3 hours in air.

As-sintered ceramic samples with the highest relative density of approximately $95 \%$ were lightly ground, coated by silver paste (Sliver PZT from Gwent group) and fired at $500^{\circ} \mathrm{C}$ for 30 mins to form electrodes. For measurement of ferroelectric properties, the specimens were poled in a silicone oil bath by applying at least 40 cycles of a sinusoidal AC electric field of $5 \mathrm{kV} \mathrm{mm}^{-1}$ at a frequency of $2 \mathrm{~Hz}$, until a stable state was achieved. Ferroelectric polarisation-electric field (P-E) and current density-electric field (J-E) loops were obtained using the method described previously. ${ }^{25}$ Microstructural examination was carried out using scanning electron microscopy (Phillip XL30) after grinding, polishing and thermally-etching at $1000{ }^{\circ} \mathrm{C}$ for 15 mins. Average grain size was estimated by linear intercept method. 
Powder-type samples were employed for high-resolution synchrotron x-ray diffraction measurements at beamline $111^{26}$, Diamond Light Source Ltd., UK. Unpoled ceramic powders were prepared by crushing and grinding as-sintered ceramic pellets by hand using a pestle and mortar, followed by annealing at $550^{\circ} \mathrm{C}$ for 30 mins. Poled ceramic powders were also prepared by a similar method from poled ceramic pellets, but without the annealing procedure. It was shown in previous publications ${ }^{23,24}$ that this procedure provides the means to identify the crystal structure of a poled sample after a field-induced transformation, as noted above. The powder samples were loaded into glass capillaries of $0.3 \mathrm{~mm}$ diameter. A photon energy of $25 \mathrm{keV}$ was selected to reduce the effect of sample absorption ( $\mu^{\prime} r \sim 2.5$. where $\mu$ is the absorption coefficient and $r$ is the diameter if the capillary sample) and the resulting diffraction patterns were recorded using a multi-analyser crystal (MAC) detectors at room temperature. The wavelength of $\lambda=0.494731(10) \AA$ was calibrated using the diffraction pattern of a high quality $\mathrm{Si}$ standard powder (SRM640c). Full-pattern refinements were carried out using Topas refinement software, v3.0. ${ }^{27}$ In addition to crystallographic parameters and structural parameters, the lattice parameters and relative phase quantity were obtained from refinements of the SXPD patterns.

\section{Results and discussion}

Prior to the diffraction experiments, an alternating electric field with amplitude of $5.5 \mathrm{kV} \mathrm{mm}^{-1}$ was applied to the NBT-0.03KN virgin sample at room temperature. It was observed that several cycles of the electric field were required in order to obtain a saturated P-E loop, as shown by the results presented in Figure 1(a). A maximum polarization $\left(P_{\max }\right)$ of $0.30 \mathrm{C} \mathrm{m}^{-2}$ was obtained after approximately 20 cycles of the electric field, remaining approximately constant thereafter. It was noted in a previous publication that a saturated P-E loop would not normally be expected for a relaxor ferroelectric such as NBT-xKN, in which the crystal structure appeared to be pseudo-cubic, ${ }^{22}$ indicating that an electric field-induced structural transformation occurred during the measurement procedure. Also, it is evident from Figure 1(b) that both the remanent and saturation polarisation values ( $P_{\text {rem }}$ and $P_{\max }$ ) increased gradually over a period of 20 cycles, suggesting that this was a progressive transformation. It was found that fewer cycles were required to obtain a stable state for higher $\mathrm{KN}$ contents, whereas at least 40 cycles were needed for $1 \% \mathrm{KN}$. 

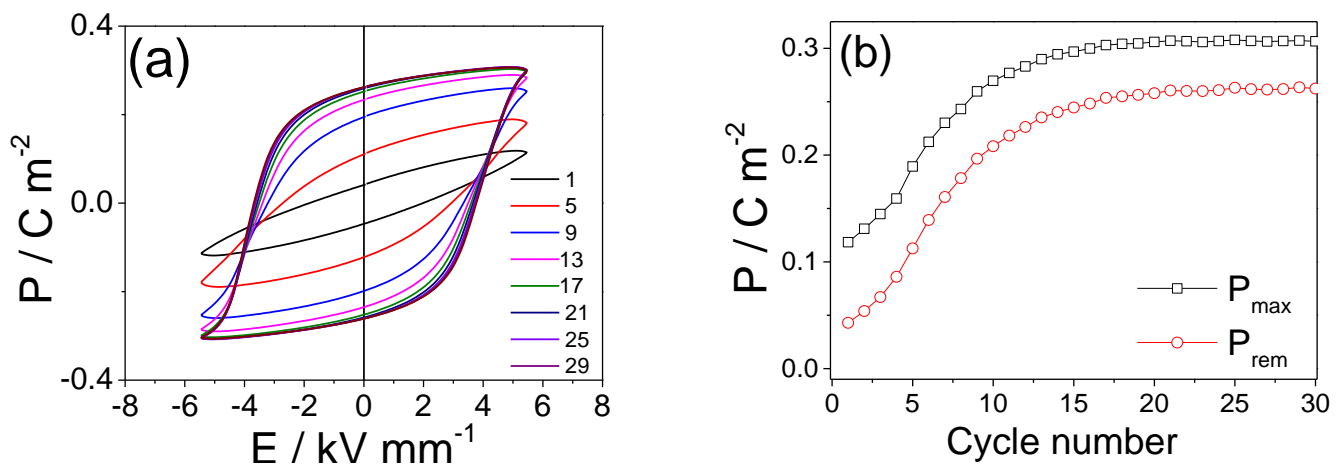

Figure 1. Changes in (a) P-E loops and (b) polarisation values obtained for NBT-0.03KN ceramics after cycling under an AC electric field.

The microstructures of the NBT-0.03KN and NBT-0.09KN ceramics are illustrated by the micrographs presented in Figure 2(a-b); the average grain sizes were determined as 2.2 $\mu \mathrm{m}$ and $1.5 \mu \mathrm{m}$ respectively. After crushing and grinding, an irregular powder was obtained which comprised individual particles and hard agglomerates with a range of sizes up to $10 \mu \mathrm{m}$, as illustrated in Figure 2(c-d). Most importantly, these particles and agglomerates are oriented randomly in the powder, which should enable a texture-free diffraction pattern to be obtained. The presence of agglomerates in the crushed powders could potentially give rise to residual stresses, which in turn could cause anisotropic broadening of the diffraction peaks. ${ }^{28}$ Inspection of the measured and calculated diffraction patterns (see discussion below) led to the conclusion that such effects were not a significant factor in the present study.
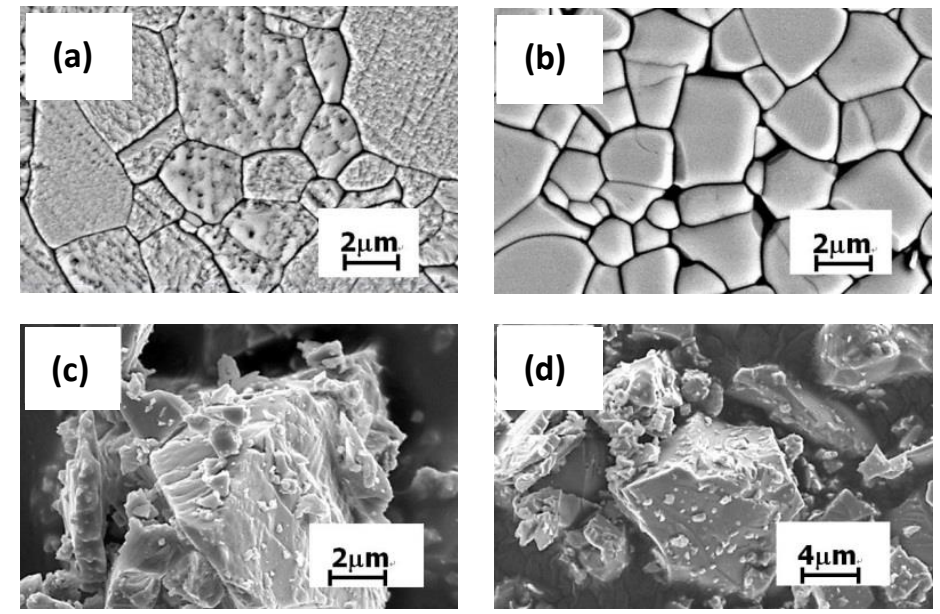

Figure 2. SEM image of (a) NBT-0.03KN ceramic cross section (b) NBT-0.09KN ceramic cross section (c) NBT-0.03KN crushed ceramic powder (d) NBT-0.09KN crushed ceramic powder

High-resolution synchrotron x-ray diffraction patterns obtained for NBT-0.03KN unpoled and poled crushed ceramic powders are illustrated in Figure 3. For the unpoled state, all reflections are single peaks, indicating the presence of a pseudo-cubic phase. In contrast, the pattern obtained for the poled NBT-0.03KN ceramic was characteristic of the rhombohedral phase with double $\{111\}_{p}$ and single $(200)_{p}$ peaks, indicating that an irreversible transformation from pseudo-cubic to rhombohedral had occurred during poling. 
Peak splitting was also evident in the doublet $\{210\}_{p}$ and triplet $\{211\}_{p}$ peaks, as shown in Figure 3.

According to these SXPD results, a rhombohedral distortion was clearly identified for the poled ceramic powders, indicating that the grinding procedure did not cause a reverse structural transformation back to the cubic phase. Similar observations were made by $\mathrm{Garg}^{23}$ and $\mathrm{RaO}^{24}$ for the NBT-BT system, as noted above.

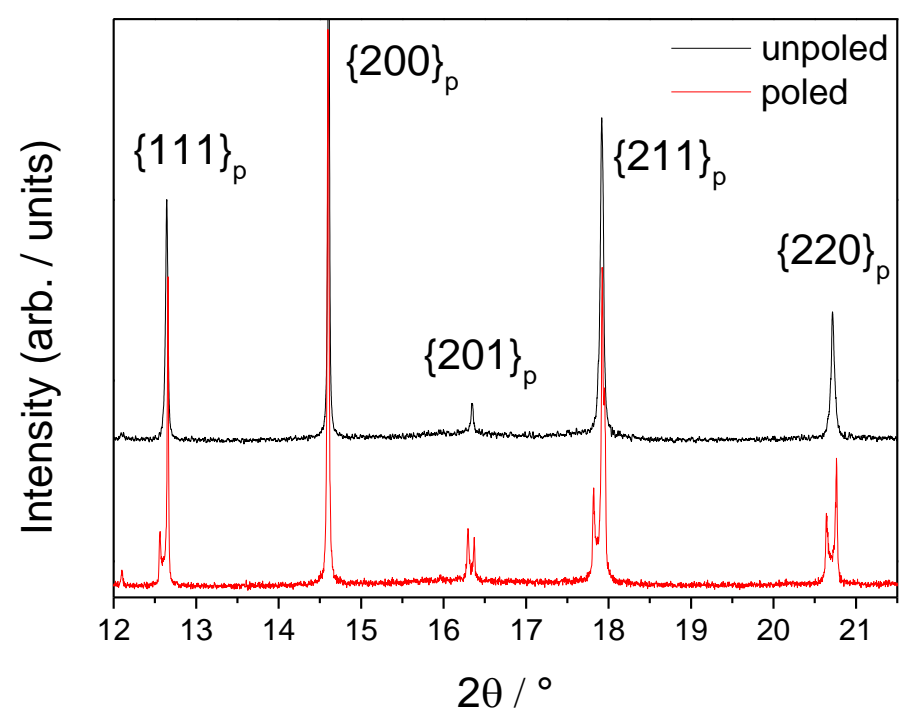

Figure 3. SXPD patterns of both unpoled and poled NBT-0.03KN powders obtained from crushed ceramic pellets.

The polarization-electric field (P-E) and current density-electric field (J-E) relationships obtained for NBT-xKN ceramics $(x=0.01,0.03,0.05$ and 0.09$)$ at room temperature after poling under an $\mathrm{AC}$ electric field of $5 \mathrm{kV} \mathrm{mm}^{-1}$ are presented in Figure 4. Typical ferroelectric behaviour was observed for NBT-0.01KN, with the peaks in the J-E curves being produced by polarization switching at the coercive field. ${ }^{29}$ However, the remanent polarization value for this composition $\left(\sim 0.08 \mathrm{C} \mathrm{m}^{-2}\right)$ was relatively low, indicating that only a poorly saturated state was obtained due to a high coercive field $\left(>4 \mathrm{kV} \mathrm{mm}^{-1}\right)$. In contrast, the NBT-0.03KN ceramic exhibited more complete ferroelectric switching behaviour at room temperature with a remanent polarization of approximately $0.24 \mathrm{C} \mathrm{m}^{-2}$, suggesting that an irreversible phase transition from weak-polar to ferroelectric-ordered phase had occurred during the measurement procedure. This type of electric field-induced transition has been reported previously by $\mathrm{Ma}^{30}$ and $\mathrm{Guo}^{31}$, who used in-situ TEM to observe a transformation from PNRs to metastable ferroelectric domain structures in NBT-BT solid solutions.

The NBT-0.05KN ceramic also yielded well-saturated ferroelectric hysteresis loops, but displayed split peaks in the J-E curve and a slight constriction in the P-E curve at room temperature. This kind of pinched loop, which has been found previously in NBT-BT-KNN, ${ }^{32}$ NBT-BT-Mn ${ }^{33}$ and NBT-BT-CaTiO ${ }_{3}^{29}$ solid solutions, has been attributed to the reversible transformation between weak-polar and ferroelectric-ordered phases. The transition in 
NBT-0.05KN was found to be partially reversible, giving rise to two peaks in the J-E curve which represent the forward electric field $\left(E_{F}\right)$ and backward electric field $\left(E_{B}\right)$ to trigger the switching. ${ }^{29}$ Therefore, it is concluded that at room temperature an irreversible transformation from weak-polar to the ferroelectric-ordered phase occurs for $\mathrm{x}=0.01$ and $x=0.03$, with the process being partially reversible for $x=0.05$. The results for $x=0.09$ show weak ferroelectric behaviour with a low remanent polarization of approximately $0.06 \mathrm{C} \mathrm{m}^{-2}$ being obtained at an electric field amplitude of $5 \mathrm{kV} \mathrm{mm}^{-1}$. The general trend of reducing remanent polarization with increasing $\mathrm{KN}$ content in NBT-KN ceramics is consistent with the reduction of depolarization temperature, as reported by Jiang et al. ${ }^{21}$
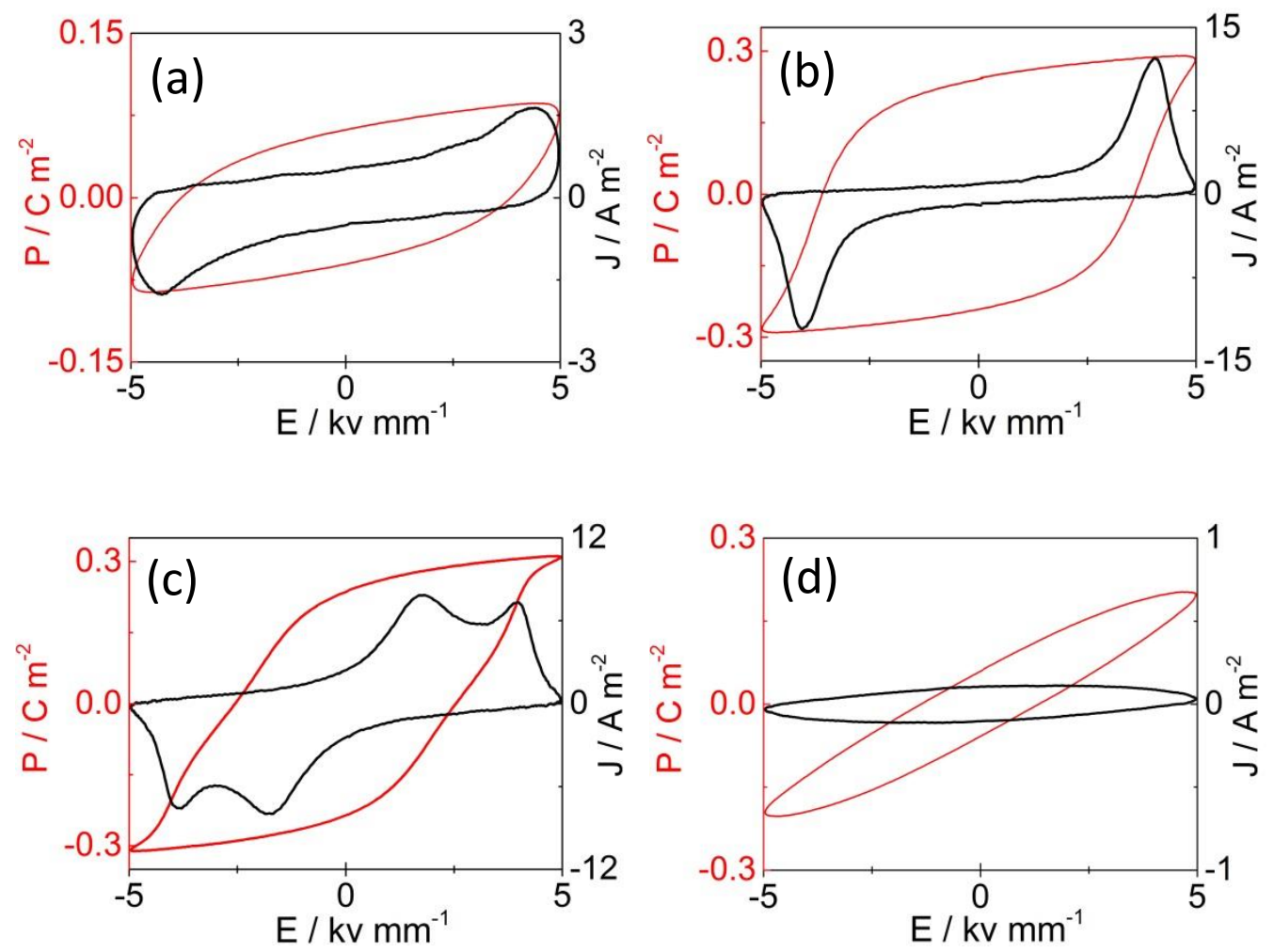

Figure 4. P-E and J-E loops of NBT-xKN ceramic pellets at room temperature measured under a maximum electric field of $5 \mathrm{kV} \mathrm{mm}^{-1}$ and frequency of $2 \mathrm{~Hz}$ for (a) $x=0.01$ (b) $x=0.03$ (c) $x=0.05$ (d) $x=0.09$

Selected regions of the $\mathrm{x}$-ray diffraction patterns obtained for both unpoled and poled ceramic powders having a range of different compositions are presented in Figure 5 (a) and (b) respectively. For the unpoled state, the powders of all compositions were found to be predominantly pseudo-cubic, characterised by single unsplit diffraction peaks for all reflections. The exception was the NBT-0.01KN powder, which was observed to exhibit broad shoulders on the lower-angle side of the diffraction peaks for $\{111\}_{p},\{211\}_{p}$ and $\{220\}_{p}$. All peaks shifted to lower angles with increasing KN content, indicating an expansion of the lattice.

In the poled state, the presence of the rhombohedral phase, characterised by a double 
$\{111\}_{p}$ peak and a single $(200)_{p}$ peak, was clearly evident for NBT-0.01KN, NBT-0.03KN and NBT-0.05KN powders. We conclude that the pseudo-cubic (nano-polar) phase transformed into a metastable rhombohedral (ordered-ferroelectric) phase during poling and was retained after crushing into powder for these compositions. The rhombohedral distortion decreased with increasing KN content, with the NBT-0.09KN specimen showing only the pseudo-cubic structure after poling.
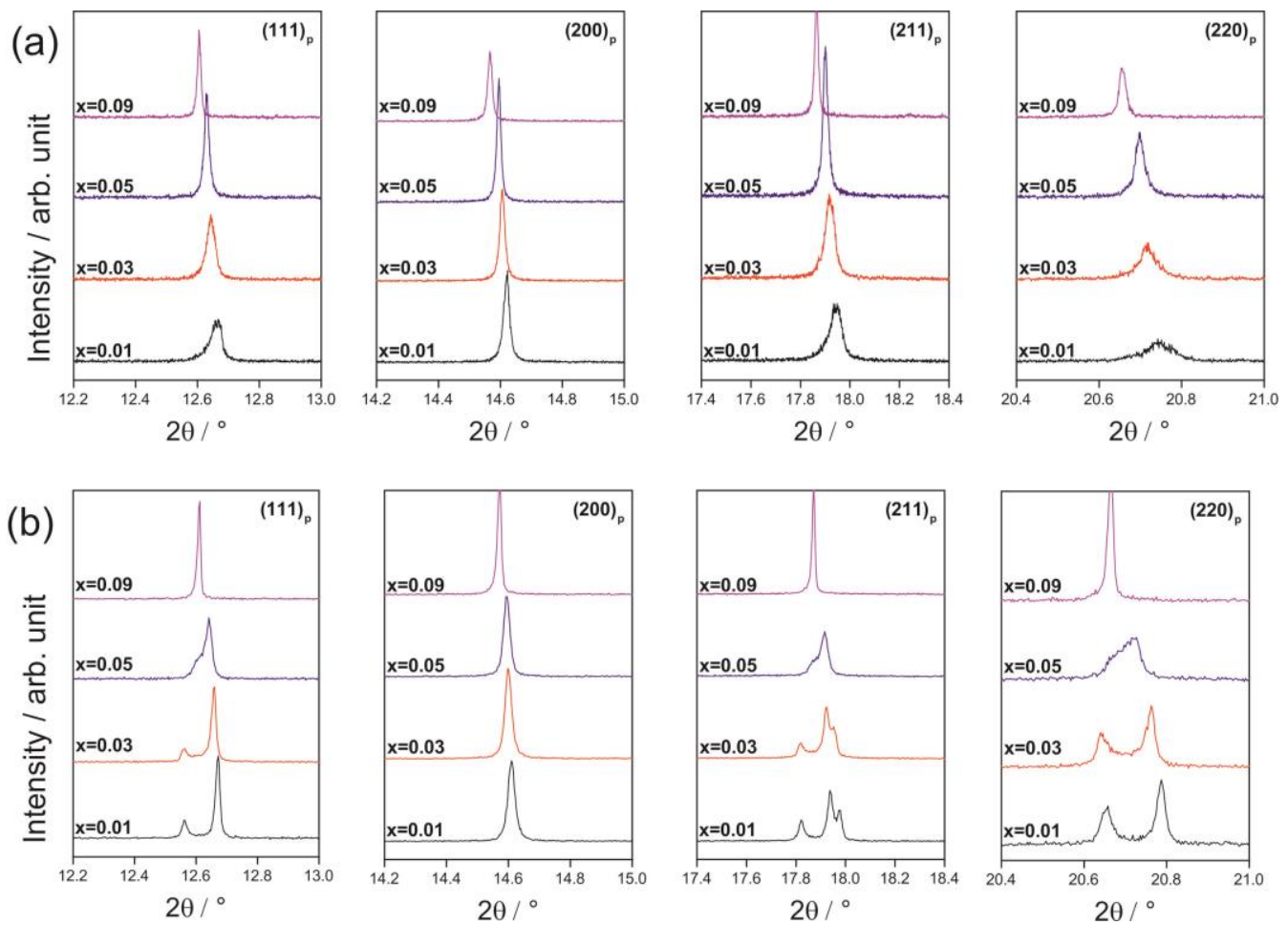

Figure 5. Selected regions of SXPD patterns illustrating $\{111\}_{p},\{200\}_{p},\{211\}_{p}$ and $\{220\}_{p}$ peaks of (a) unpoled ceramic powders and (b) poled ceramic powders for NBT-xKN solid solutions.

Full-pattern refinement of the diffractions patterns was undertaken using Topas in order to quantify the phase composition and lattice parameters of the NBT-xKN ceramics both prior to and after poling. For example, the unpoled NBT-0.03KN, which on first inspection appeared to be single phase cubic, was fitted using the R3c space group, yielding a small rhombohedral distortion (see discussion below). For the poled NBT-0.03KN powder, several different single or mixed phase structures were considered including, for example, the Cc monoclinic phase reported by Aksel. ${ }^{34}$ However, the best fit was obtained using a single phase R3c rhombohedral space group. The experimental and calculated diffraction patterns are illustrated using four representative reflections in Figure 6 . The best $R_{w p}$ values obtained for the unpoled and poled states were $6.77 \%$ and $7.24 \%$ respectively. 

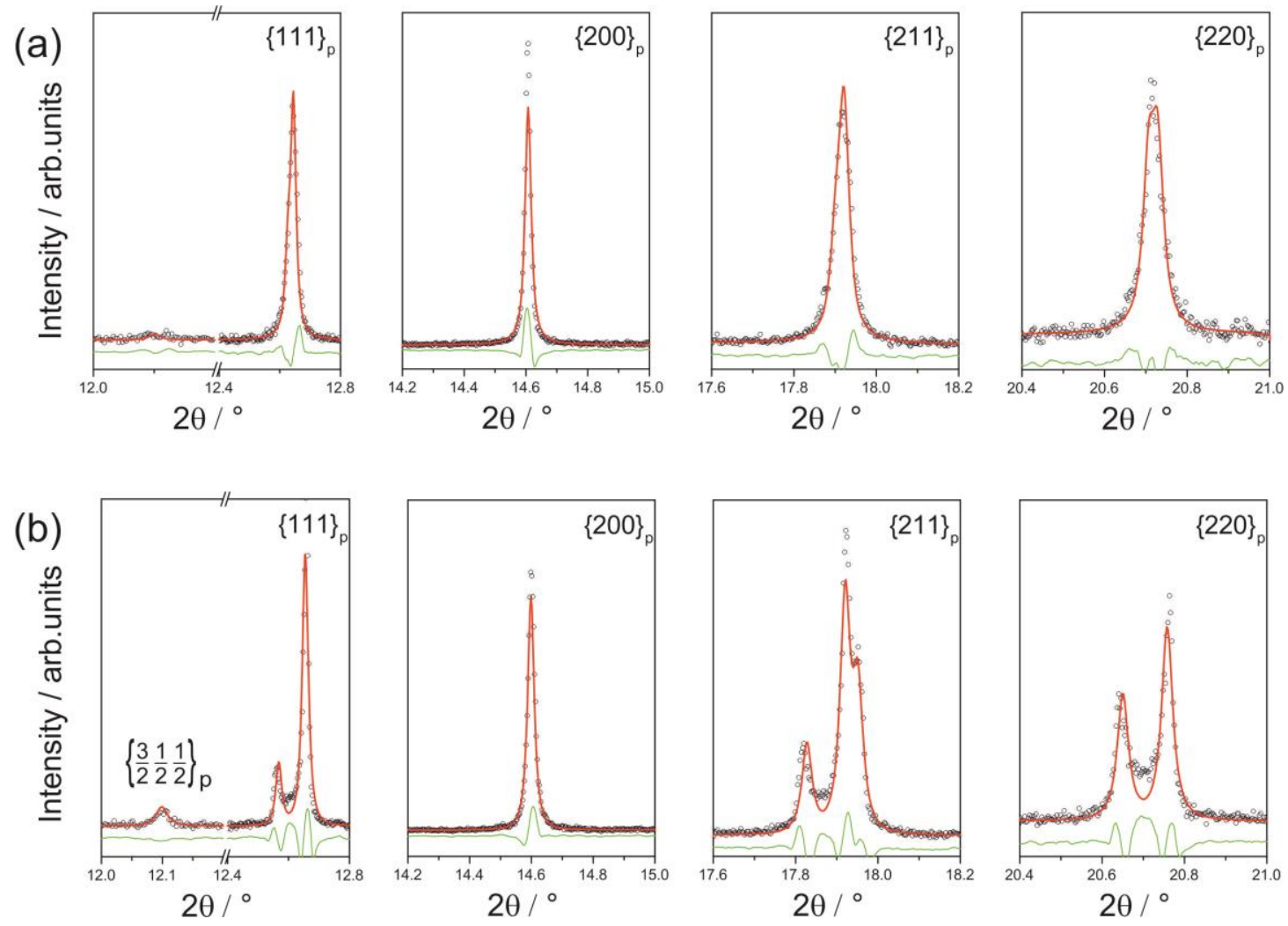

Figure 6. (a) Room-temperature SXPD patterns for (a) unpoled and (b) poled NBT-0.03KN ceramic powders. The symbols are experimental data, while the red line is the calculated pattern from Rietveld refinement using the rhombohedral R3c space group and the green line is the difference between the experimental and calculated patterns.

An additional weak diffraction peak, indexed as $\left\{\frac{3}{2} \frac{1}{2} \frac{1}{2}\right\}_{p}$, was identified for the poled specimens containing 1 to $5 \% \mathrm{KN}$, which is associated with oxygen octahedral tilting in the R3c rhombohedral phase. ${ }^{35} A$ diffuse region was apparent between the split $\{111\}_{p},\{211\}_{p}$ and $\{220\}_{p}$ peaks, which has been attributed previously to the microstrain associated with ferroelectric domain walls. ${ }^{28,36,37}$

To facilitate a comparison between the unpoled and poled states, the rhombohedral phase, which is normally indexed according to the hexagonal unit cell, can be transformed to an equivalent pseudo-cubic unit cell through the rhombohedral unit cell, using the relationships given by Megaw ${ }^{38}$ and Cullity. ${ }^{39}$ The lattice parameter, $a_{R}$, and interaxial angle, $\alpha_{R}$, were calculated using equations 1 and 2 . Note that the volume of the hexagonal unit cell is three times greater than that of the rhombohedral one. ${ }^{39}$

$$
\begin{aligned}
& a_{R}=\frac{1}{3} \sqrt{3 a_{H}^{2}+c_{H}{ }^{2}} \\
& \sin \frac{\alpha_{R}}{2}=\frac{3}{2 \sqrt{3+\left(c_{H} / \alpha_{H}\right)^{2}}}
\end{aligned}
$$


Subsequently, $a_{R}$ and $\alpha_{R}$ for the rhombohedral unit cell can be transformed into $a_{p c}$ and $\alpha_{p c}$ for the pseudo-cubic unit cell according to Equations 3 and 4. Furthermore, the volume of the rhombohedral unit cell is double that of the pseudo-cubic unit cell. ${ }^{38}$

$$
\begin{aligned}
& a_{p c}=\frac{a_{R}}{\sqrt{2}} \\
& \alpha_{p c}=90-\frac{\sqrt{3}}{2}\left(60-\alpha_{R}\right)
\end{aligned}
$$

The results obtained for the changes in lattice parameter, $\mathrm{a}_{\mathrm{pc}}$, and rhombohedral angle, $\alpha_{\mathrm{pc},}$ as a function of composition for the NBT-xKN system are illustrated in Figure 7. For KN greater than $5 \%$, the powders in the unpoled state yielded $\alpha_{\mathrm{pc}}=90^{\circ}$; for the cases of NBT-1KN and NBT-3KN a small rhombohedral distortion was evident, reflecting the slight broadening and asymmetry in certain diffraction peaks shown in Figure 5 above. The lattice parameter, $a_{p c}$ increased slightly from $3.88812 \AA$ to $3.90146 \AA$ with increasing KN content. For the poled state, $\mathrm{a}_{\mathrm{pc}}$, was found to be approximately equal to $3.90135 \AA$ in all compositions, while $\alpha_{\mathrm{pc}}$, increased gradually with increasing $\mathrm{KN}$ content showing a reduction in the rhombohedral distortion. This effect can be correlated with the improvement in ferroelectric switching behaviour illustrated in Figure 4, since the ferroelectric coercive field in perovskites is generally found to scale with the spontaneous strain. In the case of NBT-0.09KN the lattice parameters obtained for the unpoled and poled powders were approximately equal, indicating that the electric field induced no significant changes in the structure of the pseudo-cubic phase.
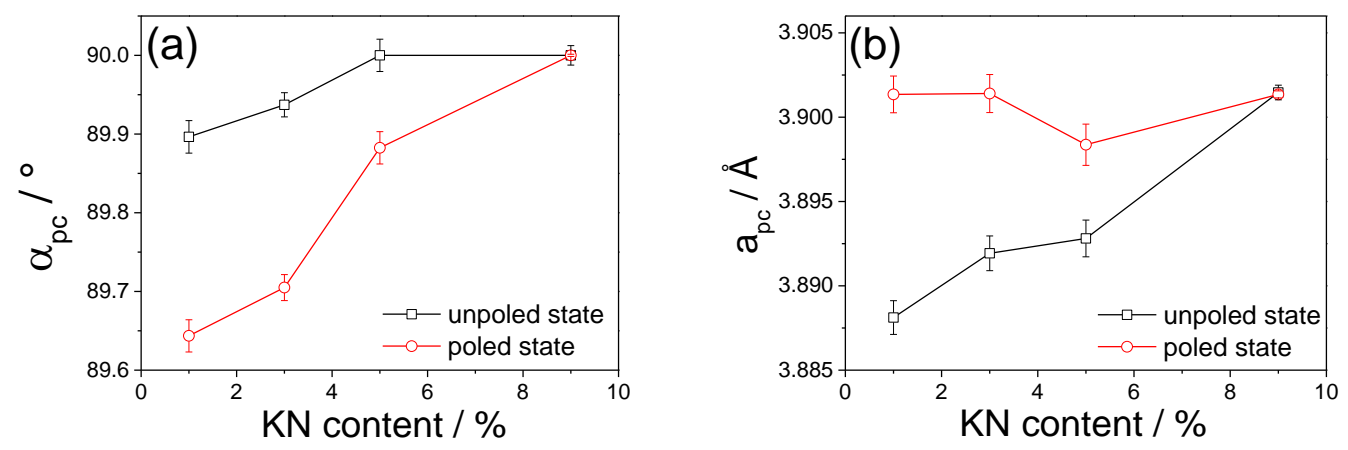

Figure 7. Comparison between unpoled and poled states for NBT-xKN powders (a) lattice parameter, $a_{p c r}$ and (b) interaxial angle, $\alpha_{p c r}$ for pseudo-cubic unit cell. 


\section{Conclusions}

The occurrence of an irreversible electric field-induced pseudo-cubic to rhombohedral phase transformation in NBT-KN ceramics was confirmed using high resolution SXPD measurements on powders produced from crushed ceramic pellets. In the unpoled state, the powders had a predominantly pseudo-cubic structure, typical of relaxor ferroelectrics. However, a small rhombohedral distortion could be detected for specimens containing $1 \%$ and $3 \% \mathrm{KN}$. In contrast, the poled powders containing $1 \%$ to $5 \% \mathrm{KN}$ exhibited a more obvious rhombohedral R3c phase, with the interaxial angle, $\alpha_{\mathrm{pc}}$ increasing from $89.6^{\circ}$ to $90^{\circ}$ with increasing $\mathrm{KN}$ content. The $9 \% \mathrm{KN}$ specimen retained the pseudo-cubic phase even after application of a high electric field. The observed variations in the rhombohedral distortion are correlated with changes in the ferroelectric polarization switching behaviour, which are affected both by the spontaneous strain, which leads to high coercive fields at low KN contents, and the disruption of the long-range ordered ferroelectric state, which causes constriction of the P-E loops at high KN contents. The study demonstrates that the analysis of crushed ceramic pellets provides a viable method to evaluate the influence of the electric field on phase content and crystal structures of ferroelectric ceramic materials.

\section{Acknowledgements}

We thank Diamond Light Source for access to beamline I11 (proposal number EE10355) that contributed to the results presented here. 


\section{References}

1. J. Rödel, W. Jo, K. T. P. Seifert, E.M. Anton, T. Granzow, and D. Damjanovic, Perspective on the Development of Lead-free Piezoceramics, J. Am. Ceram. Soc. 92 (2009) 1153-1177.

2. J. Rödel, K. G. Webber, R. Dittmer, W. Jo, M. Kimura, and D. Damjanovic, Transferring lead-free piezoelectric ceramics into application, J. Eur. Ceram. Soc. 35 (2015) 1659-1681.

3. G. A. Smolenskii, V. A. Isupov, A. I. Agranovskaya, and N. N. Krainik, New ferroelectrics of complex composition, Sov. Phys. Solid State. 2 (1961) 2651.

4. G. O. Jones and P. A. Thomas, Investigation of the structure and phase transitions in the novel A-site substituted distorted perovskite compound Na0.5Bi0.5TiO3, Acta Crystallogr. Sec. B. 58 (2002) 168-178.

5. J. Suchanicz and W. S. Ptak, On the phase transition in Na0.5Bi0.5TiO3, Ferroelectrics 12 (1990) 71-78.

6. Y. Huichun and Z. Zuo Guang, Dielectric, ferroelectric, and piezoelectric properties of the lead-free (1-x)(Na0.5Bi0.5)TiO3-xBiAlO3 solid solution, Appl. Phys. Lett. 93 (2008) 112902.

7. Y. Hiruma, Y. Imai, Y. Watanabe, H. Nagata, and T. Takenaka, Large electrostrain near the phase transition temperature of (Bi0.5Na0.5)TiO3-SrTiO3 ferroelectric ceramics, Appl. Phys. Lett. 92 (2008) 262904.

8. S.T. Zhang, A. B. Kounga, E. Aulbach, T. Granzow, W. Jo, H.-J. Kleebe, and J. Rödel, Lead-free piezoceramics with giant strain in the system Bi0.5Na0.5TiO3-BaTiO3K0.5Na0.5NbO3. I. Structure and room temperature properties, J. Appl. Phys. 103 (2008) 034107.

9. G. Fan, W. Lu, X. Wang, and F. Liang, Morphotropic phase boundary and piezoelectric properties of (Bi1/2Na1/2)TiO3 - (Bi1/2K1/2)TiO3 - KNbO3 lead-free piezoelectric ceramics, Appl. Phys. Lett. 91 (2007) 202908.

10. S.T. Zhang, A. B. Kounga, E. Aulbach, H. Ehrenberg, and J. Rödel, Giant strain in lead-free piezoceramics Bi0.5Na0.5TiO3-BaTiO3-K0.5Na0.5NbO3 system, Appl. Phys. Lett. 91 (2007) 112906.

11. A. J. Royles, A. J. Bell, A. P. Jephcoat, A. K. Kleppe, S. J. Milne, and T. P. Comyn, Electric-field-induced phase switching in the lead free piezoelectric potassium sodium bismuth titanate, Appl. Phys. Lett. 97 (2010) 132909.

12. J. E. Daniels, W. Jo, J. Rödel, and J. L. Jones, Electric-field-induced phase transformation at a lead-free morphotropic phase boundary: Case study in a 93\%(Bi0.5Na0.5)TiO3-7\%BaTiO3 piezoelectric ceramic, Appl. Phys. Lett. 95 (2009) 032904.

13. G. Shirane, H. Danner, A. Pavlovic, and R. Pepinsky, Phase Transitions in Ferroelectric KNbO3, Phys. Rev. Lett. 93 (1954) 672-673.

14. L. Liang, Y. L. Li, L.-Q. Chen, S. Y. Hu, and G.-H. Lu, Thermodynamics and ferroelectric properties of KNbO3, J. Appl. Phys. 106 (2009) 104118.

15. S. Yasuyoshi, T. Hisaaki, T. Toshihiko, N. Tatsuhiko, T. Kazumasa, H. Takahiko, N. Toshiatsu, and N. Masaya, Lead-free piezoeceramics, Nature. 432 (2004) 84-87.

16. F. Guifen, L. Wenzhong, W. Xiaohong, L. Fei, and X. Jianzhong, Phase transition behaviour and electromechanical properties of ( $\mathrm{Na} 1 / 2 \mathrm{Bi} 1 / 2) \mathrm{TiO} 3-\mathrm{KNbO} 3$ lead-free piezoelectric ceramics, J. Phys. D. 41 (2008) 035403.

17. L. Wenzhong, F. Guifen, W. Xiaohong, and L. Fei, Phase structure and electrical properties 
of Bi1/2Na1/2TiO3-KNbO3 Lead-free piezoelectric ceramics, Key Eng. Mater. 336-338 (2007) 184-187.

18. Y. Hiruma, H. Nagata, and T. Takenaka, Phase diagrams and electrical properties of (Bi1/2Na1/2)TiO3-based solid solutions, J. Appl. Phys. 104 (2008) 124106.

19. N. Pistipipathsin, W. Koontasing, S. Eitssayeam, U. Intatha, G. Rujijanagul, K. Pengpat, and T. Tunkasiri, Morphotropic Phase Boundary of Lead-free Piezoelectric Ceramics from BNT-KN System, Adv. Mater. Res. 55-57 (2008) 225-228.

20. J. Xijie, W. Baoyin, L. Laihui, L. Weiping, Z. Jun, and C. Hongbing, Electrical properties of (1-x)(Bi0.5Na0.5)TiO3-xKNbO3 lead-free ceramics, J. Solid State Chem. 213 (2014) 72-78.

21. X. Jiang, L. Luo, B. Wang, W. Li, and $\mathrm{H}$. Chen, Electrocaloric effect based on the depolarization transition in (1-x)Bi0.5Ti0.5TiO3-KNbO3 lead-free ceramics, Ceram. Int. 40 (2014) 2627-2634.

22. G. Wang, D. A. Hall, T. P. Comyn, L. Daniel, and A. K. Kleppe, Structure and ferroelectric behaviour of Na0.5Bi0.5TiO3-KNbO3 ceramics, Adv. Appl. Ceram. 115 (2016) 1-7.

23. R. Garg, B. N. Rao, A. Senyshyn, P. S. R. Krishna, and R. Ranjan, Lead-free piezoelectric system Na0.5Bi0.5TiO3-BaTiO3: Equilibrium structures and irreversible structural transformations driven by electric field and mechanical impact, Phys. Rev. B. 88 (2013) 014103. 24. B. N. Rao and R. Ranjan, Electric-field-driven monoclinic-to-rhombohedral transformation in Na1/2Bi1/2TiO3, Phys. Rev. B. 86 (2012) 134103.

25. D.A. Hall, P. J. Stevenson, and a. T. R. Mullins, Dielectric nonlinearity in hard piezoelectric ceramics, Brit. Cer. Proc. 57 (1997) 197-211.

26. S. P. Thompson, J. E. Parker, J. Potter, T. P. Hill, A. Birt, T. M. Cobb, F. Yuan, and C. C. Tang, Beamline I11 at Diamond: A new instrument for high resolution powder diffraction, Rev. Sci. Instrum. 80 (2009) 075107.

27. "Diffrac plus TOPAS v. 3.0 (Manual), BRUKER AXS GmbH, Karlsruhe, 2006.

28. D. A. Hall, A. Steuwer, B. Cherdhirunkorn, T. Mori, and P. J. Withers, A high energy synchrotron x-ray study of crystallographic texture and lattice strain in soft lead zirconate titanate ceramics, J. Appl. Phys. 96 (2004) 4245-4252.

29. G. Viola, R. McKinnon, V. Koval, A. Adomkevicius, S. Dunn, and H. Yan, Lithium-Induced Phase Transitions in Lead-Free Bi0.5Na0.5TiO3 Based Ceramics, J. Phys. Chem. C. 118 (2014) 8564-8570.

30. C. Ma, H. Guo, S. P. Beckman, and X. Tan, Creation and Destruction of Morphotropic Phase Boundaries through Electrical Poling: A Case Study of Lead-Free (Bi1/2Na1/2)TiO3-BaTiO3 Piezoelectrics, Phys. Rev. Lett. 109 (2012) 107602.

31. H. Guo, C. Ma, X. Liu, and X. Tan, Electrical poling below coercive field for large piezoelectricity, Appl. Phys. Lett. 102 (2013) 092902.

32. G. Viola, H. Ning, M. J. Reece, R. Wilson, T. M. Correia, P. Weaver, M. G. Cain, and H. Yan, Reversibility in electric field-induced transitions and energy storage properties of bismuth-based perovskite ceramics, J. Phys D. 45 (2012) 355302.

33. E. Sapper, N. Novak, W. Jo, T. Granzow, and J. Rödel, Electric-field-temperature phase diagram of the ferroelectric relaxor system $(1-\mathrm{x}) \mathrm{Bi} 1 / 2 \mathrm{Na} 1 / 2 \mathrm{TiO} 3-\mathrm{xBaTiO} 3$ doped with manganese, J. Appl. Phys. 115 (2014) 194104.

34. E. Aksel, J. S. Forrester, J. L. Jones, P. A. Thomas, K. Page, and M. R. Suchomel, Monoclinic crystal structure of polycrystalline Na0.5Bi0.5TiO3, Appl. Phys. Lett. 98 (2011) 152901. 
35. W. Jo, J. E. Daniels, J. L. Jones, X. Tan, P. A. Thomas, D. Damjanovic, and J. Rödel, Evolving morphotropic phase boundary in lead-free (Bi1/2Na1/2)TiO3-BaTiO3 piezoceramics, J. Appl. Phys. 109 (2011) 014110.

36. B. Noheda, J. A. Gonzalo, L. E. Cross, R. Guo, S. E. Park, D. E. Cox, and G. Shirane, Tetragonal-to-monoclinic phase transition in a ferroelectric perovskite: The structure of PbZr0.52Ti0.4803, Phys. Rev. B. 61 (2000) 8687-8695.

37. B. Noheda, D. E. Cox, G. Shirane, R. Guo, B. Jones, and L. E. Cross, Stability of the monoclinic phase in the ferroelectric perovskite PbZr1-xTixO3, Phys. Rev. B. 63 (2000) 014103. 38. H. D. Megaw and C. N. W. Darlington, Geometrical and structural relations in the rhombohedral perovskites, Acta Crystallogr. Sec. A. 31 (1975) 161-173.

39. B. Culity and S. Stock, Elements of X-ray Diffraction 2nd, Addition-Wesley, MA, London, (1978). 